\title{
Perspectivas da evolução clínica de pacientes com cardiomiopatia chagásica listados em prioridade para o transplante cardíaco
}

\author{
Clinical perspectives of patients with Chagas cardiomyopathy listed as high priority for heart \\ transplantation
}

Luiz Felipe P. MOREIRA, João GALANTIER, Anderson BENÍCIO, Adolfo A. LEIRNER, Alfredo I. FIORELLI, Noedir A.G. STOLF, Sérgio A. de OLIVEIRA

\section{Resumo}

Introdução: O choque cardiogênico é responsável por elevados índices de mortalidade na fila de espera para o transplante cardíaco. Na cardiomiopatia chagásica, a alta incidência de disfunção biventricular pode contribuir com a gravidade desta complicação.

Método: Foram estudados 141 pacientes indicados em caráter de prioridade para o transplante. Destes pacientes, 46 eram portadores de cardiomiopatia chagásica e 95 de outras cardiomiopatias. O choque cardiogênico foi tratado farmacologicamente e com o implante ocasional do balão intra-aórtico. Em cinco pacientes chagásicos, foi realizado o implante de dispositivo paracorpóreo de assistência ventricular esquerda.

Resultados: Num período médio de 2,8 meses, 58 (41,1\%) dos 141 pacientes foram transplantados, $73(51,7 \%)$ faleceram e $\mathbf{1 0}$ foram retirados da fila. A mortalidade entre os pacientes chagásicos e não chagásicos foi de $45,6 \%$ e $54,7 \%$, respectivamente. No entanto, a expectativa média de vida, sem a realização do transplante cardíaco, dos pacientes chagásicos foi de apenas 1,5 meses, sendo observado risco relativo de mortalidade de 1,6 para estes pacientes em relação aos não chagásicos $(\mathbf{p}<\mathbf{0 , 0 5})$. Os cinco pacientes chagásicos submetidos ao implante do dispositivo de assistência circulatória foram mantidos por um período médio de 22 dias, sendo que dois foram transplantados, dois faleceram por falência de múltiplos órgãos e um ainda está sob assistência. Nenhum destes pacientes apresentou disfunção do ventrículo direito, não tendo ocorrido qualquer complicação relacionada ao dispositivo.

Conclusão: A evolução do choque cardiogênico parece ser mais rápida na cardiomiopatia chagásica, sendo importante a indicação precoce de dispositivos de assistência mecânica como ponte para a realização do transplante cardíaco.

Descritores: Miocardiopatia chagásica. Choque cardiogênico. Transplante de coração. Coração auxiliar.

Trabalho realizado pela Unidade Cirúrgica de Pesquisa e pelo Centro de Tecnologia Biomédica do Instituto do Coração do Hospital das Clínicas da Faculdade de Medicina da Universidade de São Paulo. Estudo realizado com o apoio da FAPESP - Projeto 04/06778-4. 


\section{Abstracts}

Introduction: Heart failure is responsible for high mortality rates of patients on heart transplantation waiting lists. In Chagas cardiomyopathy, the presence of biventricular dysfunction increases the severity of this situation.

Method: One hundred and forty-one patients suffering from cardiogenic shock, listed as high priority for heart transplantation, were studied. Forty-six patients presented with Chagas cardiomyopathy and 95 with other cardiomyopathies. Heart failure was treated using intravenous inotropic drugs and intra-aortic balloon pump implantation. Five patients with Chagas disease underwent paracorporeal left ventricular assist device implantation.

Results: During a mean follow-up of 2.8 months, $58(41.1 \%)$ of the 141 patients were transplanted, while $73(53.7 \%)$ died and 10 were removed from the waiting list. The mortality rates in chagasic and non-chagasic patients were $45.6 \%$ and $54.7 \%$, respectively. The mean expected survival of patients

\section{INTRODUÇÃO}

O transplante cardíaco enfrenta atualmente o grave problema da escassez de doadores. Estima-se que entre 10\% e $40 \%$ dos pacientes selecionados falecem na fila de espera em todo o mundo e uma parcela significativa desses pacientes morre por falência circulatória progressiva [1,2]. Para esses pacientes, a utilização de dispositivos de assistência circulatória mecânica é, muitas vezes, a única possibilidade de sobrevivência durante a espera do doador.

Vários tipos de dispositivos têm sido empregados para servir como ponte para a posterior realização do transplante cardíaco e a experiência internacional neste campo já soma milhares de casos [3-6]. Com base nesta experiência, os critérios de indicação da assistência circulatória estão bem estabelecidos, assim como o impacto da aplicação desta terapêutica na expectativa de vida dos pacientes.

A cardiomiopatia chagásica é uma das principais indicações para o transplante cardíaco em nosso país, sendo uma afecção de elevada mortalidade nas fases mais avançadas de comprometimento miocárdico [7,8]. Por outro lado, apesar dos bons resultados apresentados com o emprego do transplante cardíaco no tratamento da cardiomiopatia chagásica $[9,10]$, um número elevado de pacientes portadores desta afecção evolui em choque cardiogênico, geralmente resultante de um comprometimento biventricular, vindo a falecer durante a espera de um doador. A este respeito, devido à falta de experiência com o emprego de dispositivos de assistência circulatória mecânica em nosso país, não existem estudos avaliando o impacto do adequado controle terapêutico destes pacientes durante o período de espera para o transplante. with Chagas disease, who did not undergo heart transplantation, was only 1.5 months, with these patients presenting a relative risk for death of 1.6 compared to patients with other heart diseases $(p<0.05)$. The five chagasic patients submitted to left ventricular assist device implantation were maintained on support for a mean of 22 days, with two of them undergoing transplantation, two died due to multiple organ failure and one remains on circulatory support. None of these patients presented right ventricular dysfunction and there were no device related complications.

Conclusion: The evolution of heart failure seems to be rapid in patients with Chagas cardiomyopathy. Therefore, an early indication of mechanical circulatory support is important as a bridge to heart transplantation in these patients.

Descriptors: Chagas cardiomyopathy. Shock, cardiogenic. Heart transplantation. Heart assist devices.

O objetivo deste trabalho é analisar a evolução dos pacientes portadores de cardiomiopatia chagásica que apresentam períodos de choque cardiogênico na fila de espera para o transplante cardíaco. Paralelamente, será relatada a experiência inicial com o emprego de um dispositivo paracorpóreo de assistência ventricular esquerda no tratamento destes pacientes.

\section{MÉTODO}

\section{Casuística}

No período de janeiro de 1998 a março de 2005, o transplante cardíaco foi indicado em 256 pacientes, com idade variando entre 10 e 69 anos, no Instituto do Coração. As indicações para o transplante foram a cardiomiopatia dilatada idiopática em 100 (39\%) pacientes, a cardiomiopatia chagásica em 68 (26,5\%), a cardiomiopatia isquêmica em 62 $(24,2 \%)$ e outras etiologias em 26 (10,3\%) doentes. Cento e quarenta e um $(54,8 \%)$ pacientes entraram na fila para o transplante em caráter de prioridade, por estarem em uso hospitalar de drogas inotrópicas endovenosas para tratamento de choque cardiogênico. Destes pacientes, 46 eram portadores de cardiomiopatia chagásica, constituindose no grupo de interesse deste estudo.

\footnotetext{
Manuseio da falência circulatória na fila de espera para o transplante

Todos os pacientes que evoluíram com diagnóstico de choque cardiogênico na fila de espera para o transplante foram submetidos a suporte farmacológico endovenoso. Este suporte constituiu-se em primeira instância pelo

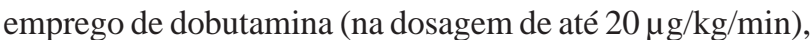


seguindo-se, quando necessário, a associação de outros fármacos, como milrinone, dopamina ou noradrenalina. A indicação da inserção de balão intra-aórtico foi realizada nos pacientes que se apresentavam refratários à terapêutica farmacológica.

A partir de outubro de 2003, foi iniciado o programa de ponte para o transplante cardíaco com o implante de dispositivo paracorpóreo de assistência ventricular. Este programa foi aprovado pela Comissão Científica e de Ética do Instituto do Coração e pelo Conselho Nacional de Pesquisa, tendo sido obtida a assinatura de Consentimento Informado Livre e Esclarecido pelos pacientes ou responsáveis legais. O implante deste dispositivo foi indicado em pacientes que apresentavam choque cardiogênico refratário ao uso da terapêutica medicamentosa e ao controle adequado da volemia. Nesta situação, os seguintes fatores estão relacionados a mau prognóstico pós-operatório e foram considerados contra-indicações para o procedimento: idade superior a 65 anos; peso menor do que 40 kg; episódio de embolia pulmonar no último mês; intubação prolongada (período maior do que 48 horas); episódio de reanimação cardiopulmonar nas últimas 24 horas; seqüela neurológica aguda; insuficiência renal aguda ou crônica, com creatinina acima de 2,5 mg/dl e/ou uréia acima de $100 \mathrm{mg} / \mathrm{dl}$; disfunção hepática com bilirrubinas totais acima de $3 \mathrm{mg} / \mathrm{dl}$; quadro infeccioso ativo ou distúrbios hemorrágicos.

A escolha do tipo de assistência circulatória (ventricular esquerda ou biventricular) foi feita de acordo com os critérios hemodinâmicos de definição de falência ventricular esquerda e direita. Foi sempre inicialmente tentado o implante isolado de um sistema de assistência ventricular esquerda, sendo a disfunção ventricular direita manuseada farmacologicamente.

\section{Implante e acompanhamento do dispositivo de assistência ventricular}

O dispositivo de assistência ventricular empregado neste estudo foi o modelo paracorpóreo InCor, desenvolvido pelo Centro de Tecnologia Biomédica do Instituto do Coração (Figura 1). O implante das cânulas e do dispositivo de assistência ventricular InCor foi realizado com o emprego de circulação extracorpórea convencional, em normotermia. Após esternotomia mediana, foram inicialmente preparadas as incisões cutâneas abdominais, através das quais são exteriorizadas as cânulas. A anastomose do enxerto tubular da cânula arterial com a aorta ascendente ou com o tronco da artéria pulmonar foi realizada de maneira término-lateral após a instituição da anticoagulação total, sob o uso da circulação extracorpórea. A inserção e sutura da cânula ventricular na ponta do ventrículo esquerdo ou na parede livre do ventrículo direito foram sempre realizadas sob suporte circulatório, procedendo-se a seguir à conexão das cânulas com o dispositivo e à retirada de ar do sistema.

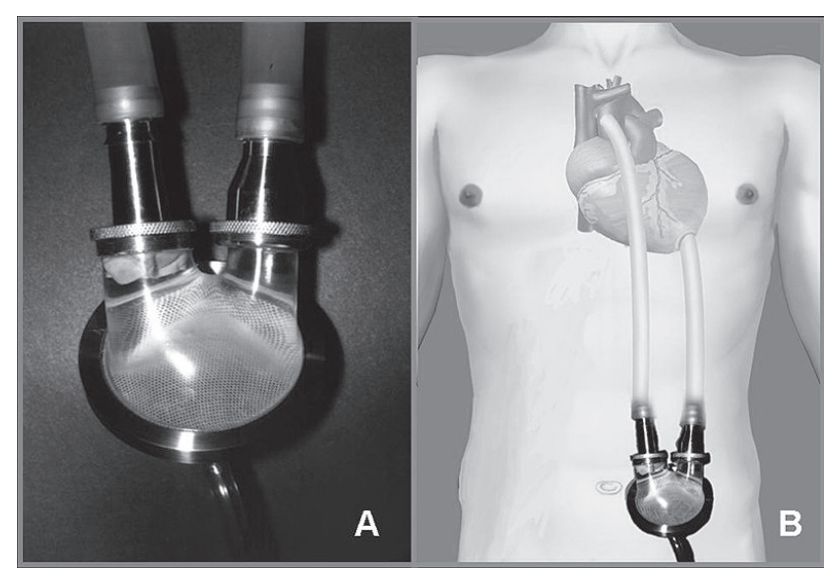

Fig. 1 - Fotografia (A) e representação esquemática (B) do dispositivo paracorpóreo de assistência ventricular InCor, implantado como suporte circulatório em paralelo aos ventrículos direito e esquerdo

Após o implante do dispositivo, os pacientes foram mantidos internados em Unidade de Terapia Intensiva durante as duas primeiras semanas de pós-operatório, sendo transferidos para Unidade semi-intensiva durante o seguimento tardio. Nos pacientes mantidos em assistência ventricular esquerda isolada, a monitorização hemodinâmica foi realizada através da inserção de cateter de Swan-Ganz, nos primeiros dias de seguimento pós-operatório. Durante todo o período de acompanhamento pós-implante, o dispositivo de assistência ventricular proporciona a monitorização contínua do débito sangüíneo através do aparelho.

Durante a assistência ventricular esquerda, o fluxo do dispositivo deve ser sempre superior a $2,5 \mathrm{l} / \mathrm{min} / \mathrm{m}^{2}$. Paralelamente, deve se manter a pressão de átrio esquerdo ou a pressão capilar pulmonar entre 5 e 15 mmHg. Na assistência biventricular, as mesmas medidas anteriormente descritas devem ser mantidas em relação ao dispositivo de assistência ventricular esquerda, sendo importante a manutenção do fluxo do dispositivo de assistência ventricular direita sempre $10 \%$ abaixo do dispositivo à esquerda.

O diagnóstico de falência ventricular direita foi estabelecido de acordo com critérios hemodinâmicos e ecocardiográficos. Todos os pacientes foram submetidos à prevenção desta complicação nos primeiros dias de seguimento, por meio da manutenção de suporte inotrópico endovenoso e de vasodilatadores pulmonares, destacandose o emprego rotineiro de óxido nítrico inalatório. Na presença de disfunção do ventrículo direito, o suporte farmacológico foi intensificado com o emprego associado de drogas inotrópicas endovenosas e de vasodilatadores pulmonares, 
como o milrinone, a nitroglicerina, e a prostaciclina. O implante de um sistema de assistência ventricular direita deve ser apenas realizado nos casos que apresentem refratariedade ao tratamento farmacológico otimizado.

\section{Análise estatística}

Os índices de sobrevivência dos pacientes na ausência do transplante cardíaco foram calculados através do método de Kaplan-Meier e apresentados com intervalo de confiança de 95\%. As curvas de sobrevivência obtidas foram comparadas entre si através do teste de log-rank. O teste de qui-quadrado foi empregado na comparação das proporções observadas. As medidas hemodinâmicas obtidas nos pacientes submetidos à assistência circulatória foram comparadas através do teste não paramétrico de Friedman. O nível de significância estabelecido foi 5\%.

\section{RESULTADOS}

Dos 256 pacientes que entraram na fila de espera para o transplante cardíaco no Instituto do Coração, 112 (43,7\%) foram submetidos ao transplante, 105 (41\%) faleceram na fila de espera, 27 (10,5\%) foram retirados da fila por melhora clínica ou por outras complicações e 12 (4,7\%) permanecem à espera do doador. A incidência de mortalidade na fila de espera foi mais elevada para os 141 pacientes que estiveram na fila de espera para o transplante em caráter de prioridade (51,7\%), enquanto que o transplante cardíaco foi realizado apenas em $41,1 \%$ destes casos. Paralelamente, a expectativa de vida destes pacientes sem a realização do transplante cardíaco foi de apenas 66,6 \pm 8,3\% em 1 mês de seguimento, de 51,6 \pm 9,5\% em 2 meses e de 35,7 $\pm 11 \%$ em 6 meses, como mostra a Figura 2.

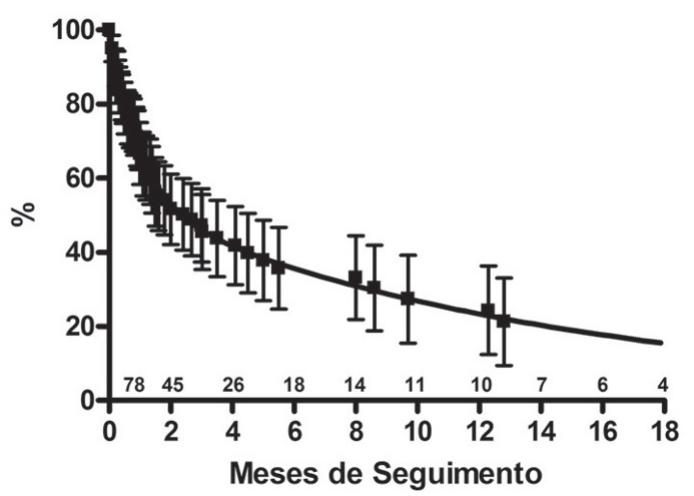

Fig. 2 - Curva de expectativa de vida dos pacientes listados em caráter de prioridade para o transplante cardíaco. Os números indicam os pacientes em risco nos períodos assinalados (média \pm IC 95\%)
Em relação à cardiomiopatia chagásica, 37 (54,4\%) dos 68 pacientes listados foram submetidos ao transplante, enquanto que 27 (39,7\%) faleceram na fila de espera e apenas três $(4,4 \%)$ foram retirados da fila por melhora clínica. Por outro lado, a ocorrência de indicações para o transplante cardíaco em caráter de prioridade foi significativamente mais elevada para os pacientes com cardiomiopatia chagásica $(67,6 \%)$ do que para os pacientes com cardiomiopatias de outras etiologias $(50,5 \%)(p=0,026)$, sendo observado um risco relativo de 1,31 (1,05 a 1,634, IC 95\%) nesta situação.

A Figura 3 mostra que a expectativa de vida dos pacientes chagásicos listados para o transplante cardíaco em caráter de prioridade foi menor do que a observada para os pacientes indicados nesta condição por causa de outras afecções. Para os pacientes portadores de cardiomiopatia chagásica, a expectativa de vida sem o transplante cardíaco foi de 57,9 $\pm 16,3 \%$ em 1 mês de seguimento, de 41,8 \pm 19,6\% em 2 meses e de 5,2 $\pm 21 \%$ em 6 meses. Paralelamente, os índices de expectativa de vida para os pacientes de outras etiologias foram de 70,3 \pm 9,6\%, 55,2 $\pm 10,9 \%$ e de $38,6 \pm 12,4 \%$ nos mesmos períodos, respectivamente. Estes dados podendo também ser expressos por uma razão de risco de mortalidade de 1,606 (1,041 a 3,304, IC 95\%) para os pacientes chagásicos em relação aos não chagásicos, sendo observada uma sobrevivência média de apenas 1,5 mês para estes pacientes.

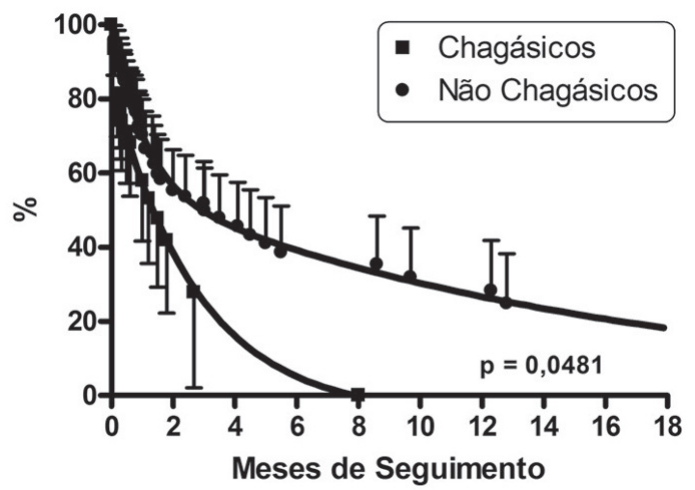

Fig. 3 - Curva de expectativa de vida dos pacientes chagásicos e não chagásicos listados em caráter de prioridade para o transplante cardíaco (média \pm IC 95\%)

Quando são analisadas as causas que conduziram ao óbito de 73 pacientes listados para o transplante cardíaco em caráter de prioridade, pode-se constatar que 23 (31,5\%) pacientes faleceram por complicações que foram responsáveis pela sua retirada da fila antes do evento final e da indicação de suporte circulatório. Outros 10 (13,5\%) pacientes faleceram tardiamente, quando o quadro de falência circulatória responsável pela indicação em 
prioridade havia sido revertido. Trinta e seis $(49,3 \%)$ pacientes evoluíram com refratariedade ao emprego otimizado do suporte inotrópico endovenoso e em alguns casos à inserção do balão intra-aórtico. Estes pacientes constituem o grupo de pacientes potencialmente elegíveis para o implante de dispositivos de assistência circulatória mecânica, ao lado dos seis pacientes que foram submetidos a este tipo de intervenção a partir de outubro de 2003.

Dos seis pacientes que receberam o implante do dispositivo paracorpóreo de assistência ventricular InCor, cinco apresentavam choque cardiogênico secundário à cardiomiopatia de etiologia chagásica. Todos os pacientes haviam entrado na fila de espera para o transplante já em caráter de prioridade e apresentavam sinais de refratariedade ao tratamento farmacológico ou ao uso de balão intra-aórtico, no momento da indicação do dispositivo de assistência circulatória. Nenhum deles estava sob assistência ventilatória mecânica ou apresentava qualquer contraindicação para o procedimento. Os outros dados préoperatórios destes pacientes são descritos na Tabela 1.

Os cinco pacientes foram submetidos ao implante de dispositivo de assistência ventricular esquerda isolada, sob circulação extracorpórea. O emprego de suporte farmacológico endovenoso, com dobutamina e milrinone, foi associado ao uso de óxido nítrico inalatório, em todos os pacientes, com o objetivo de controlar a disfunção das câmaras direitas no período pós-operatório imediato. Do ponto de vista hemodinâmico, houve manutenção de um fluxo adequado através do dispositivo de assistência ventricular esquerda durante todo o período pós-operatório, proporcionando a manutenção de índices cardíacos sempre acima de 2,51/min/ $\mathrm{m}^{2}$. Esta condição ocorreu associada à queda progressiva das pressões em câmaras direitas nos pacientes, como mostra a Figura 4. A queda das pressões venosas foi observada nestes pacientes na presença de doses progressivamente menores de medicações inotrópicas endovenosas.

Foi possível a retirada do suporte ventilatório nos cinco pacientes entre o primeiro e o quarto dia de seguimento. Este fato possibilitou a realimentação e a deambulação precoce dos mesmos, situação que foi mantida até a ocorrência de complicações que motivaram a descontinuidade destas funções nos dois pacientes que faleceram em uso da assistência circulatória.

Os resultados do implante do dispositivo de assistência ventricular como ponte para o transplante cardíaco nos cinco pacientes são apresentados na Tabela 2. Apesar das complicações relacionadas às alterações da coagulação, a evolução das funções renal e hepática foi sempre normal nos dois pacientes com maior tempo de seguimento. Nos outros dois pacientes que evoluíram com complicações pulmonares e infecciosas, houve piora tardia das funções renal e hepática, fato que colaborou com o quadro final de falência de múltiplos órgãos.

Não ocorreram complicações tromboembólicas ou relacionadas ao dispositivo de assistência circulatória em nenhum dos pacientes estudados. Paralelamente, não foram observadas alterações importantes nas superfícies internas e nas válvulas dos ventrículos artificiais por ocasião de sua retirada. Os dois pacientes que faleceram em assistência foram submetidos a estudo anátomo-patológico, que também não evidenciou qualquer sinal de fenômenos tromboembólicos nos órgãos investigados.

Tabela 1. Características pré-operatórias dos pacientes submetidos ao implante de dispositivo paracorpóreo de assistência circulatória.

\begin{tabular}{|c|c|c|c|c|c|c|c|}
\hline & Sexo & Idade & Peso (kg) & $\begin{array}{l}\text { Tipo de Suporte } \\
\text { Farmacológico }\end{array}$ & $\begin{array}{l}\text { Tempo } \\
\text { de Uso }\end{array}$ & $\begin{array}{c}\text { Índice Cardíaco } \\
(1 / \mathrm{min} / \mathrm{m} 2)\end{array}$ & $\begin{array}{l}\text { Resist. Vasc. } \\
\text { Pulm. (Wood) }\end{array}$ \\
\hline Pac.1 & Fem. & 48 & 66 & $\begin{array}{l}\text { Dobutamina } \\
\text { Nor-adrenalina }\end{array}$ & 2 dias & 1,47 & 1,72 \\
\hline Pac.2 & Masc. & 46 & 60 & $\begin{array}{c}\text { Dobutamina } \\
\text { BIA }\end{array}$ & 28 dias & 1,53 & 2.68 \\
\hline Рac.3 & Masc. & 47 & 75 & $\begin{array}{c}\text { Dobutamina } \\
\text { BIA }\end{array}$ & 12 dias & 1,87 & 2,34 \\
\hline Pac. 4 & Masc. & 52 & 85 & $\begin{array}{c}\text { Dobutamina } \\
\text { Milrinone } \\
\text { BIA }\end{array}$ & 81 dias & 1,69 & 3,11 \\
\hline Pac. 5 & Masc. & 33 & 57 & $\begin{array}{c}\text { Dobutamina } \\
\text { Milrinone } \\
\text { BIA }\end{array}$ & 25 dias & 1,72 & 1,9 \\
\hline
\end{tabular}

BIA = Balão intra-aórtico 
(A) Índice Cardíaco (Fluxo DAV)

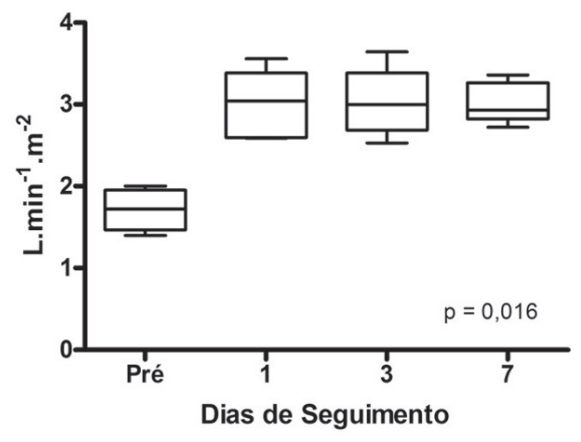

(B) Pressão Venosa Central

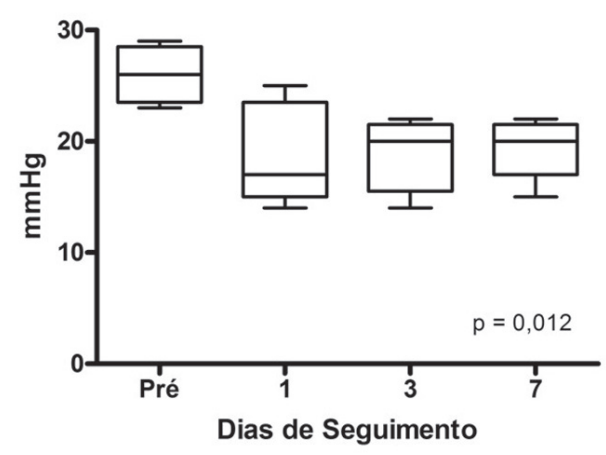

Fig. 4 - Valores do fluxo mantido através do dispositivo de assistência ventricular esquerda (A) e da pressão venosa central (B), durante a assistência circulatória nos cinco pacientes

\section{COMENTÁRIOS}

Este estudo demonstrou que a cardiomiopatia chagásica é uma afecção que cursa com elevado risco de mortalidade entre os pacientes com indicação de transplante cardíaco, em decorrência da alta incidência de choque cardiogênico e de sua curta evolução para o óbito na ausência de mecanismos adequados de suporte circulatório. Neste sentido, o emprego de dispositivos mecânicos de assistência circulatória como ponte para o transplante cardíaco abre novas perspectivas para os pacientes portadores desta afecção, propiciando aumento expressivo das chances de realização do transplante e a diminuição dos índices de mortalidade na fila de espera.

Estudos anteriores já demonstraram que a expectativa de vida de pacientes portadores de cardiomiopatia chagásica e sintomas importantes de insuficiência cardíaca é mais limitada do que a observada para pacientes com cardiomiopatias de outras etiologias [7,8]. Este fato parece estar relacionado à ocorrência freqüente de disfunção biventricular e de arritmias ventriculares complexas nos pacientes chagásicos [7,11]. No entanto, apesar da gravidade do comprometimento miocárdico ser maior na presença desta afecção, os pacientes encaminhados para o transplante cardíaco são mais jovens, sendo observados com este procedimento resultados que se sobrepõem aos observados na presença de outras indicações [9].

Por outro lado, o número elevado de pacientes chagásicos que necessitam da realização do transplante

Tabela 2. Resultados do implante de dispositivo paracorpóreo de assistência circulatória como ponte para o transplante cardíaco

\begin{tabular}{|c|c|c|c|c|}
\hline & $\begin{array}{l}\text { Tempo de } \\
\text { Assistência }\end{array}$ & Complicações & $\begin{array}{l}\text { Número de } \\
\text { Reoperações }\end{array}$ & $\begin{array}{l}\text { Resultado } \\
\text { Final }\end{array}$ \\
\hline Pac. 1 & 11 dias & $\begin{array}{l}\text { Coagulopatia; } \\
\text { Embolia pulmonar; } \\
\text { Falência de múltiplos órgãos. }\end{array}$ & 1 & Óbito \\
\hline Pac. 2 & 21 dias & Coagulopatia; Sangramento cirúrgico. & 3 & Transplante \\
\hline Pac.3 & 31 dias & Coagulopatia; Sangramento cirúrgico. & 2 & Transplante \\
\hline Pac. 4 & 18 dias & $\begin{array}{l}\text { Infecção pulmonar; } \\
\text { Falência de múltiplos órgãos. }\end{array}$ & - & Óbito \\
\hline Pac. 5 & 38 dias & $\begin{array}{l}\text { Insuficiência renal; } \\
\text { Sangramento digestivo; } \\
\text { Infecção pulmonar. }\end{array}$ & - & Em assistência \\
\hline
\end{tabular}


cardíaco em caráter de prioridade e a alta mortalidade, observada na fila de espera para estes pacientes, mostram a importância do emprego de dispositivos de assistência circulatória mecânica como ponte para o transplante cardíaco nesta afecção. Apesar da ampla experiência internacional com este tipo de procedimento, existe apenas um relato anterior de paciente portador de doença de Chagas submetido a implante de dispositivo paracorpóreo de suporte ventricular antes da realização do transplante [12]. Este relato se refere ao emprego pioneiro de um modelo inicial do dispositivo atualmente em uso nesta experiência, que proporcionou suporte circulatório esquerdo durante quatro dias.

Os critérios de indicação de dispositivos de assistência circulatória como ponte para o transplante, na experiência internacional, incluem geralmente pacientes em fases mais precoces de desenvolvimento da falência ventricular [3,5,6]. Esta situação é motivada pela influência negativa do comprometimento de outros órgãos nos resultados do suporte circulatório. A este respeito, a observação neste estudo de que o óbito entre os pacientes listados para o transplante em caráter de prioridade ocorre mais precocemente nos pacientes chagásicos, também reforça a necessidade da indicação mais precoce de dispositivos de assistência nesta afecção.

O emprego de dispositivos de assistência circulatória mecânica como ponte para o transplante cardíaco soma atualmente milhares de casos, os quais demonstram o seu indiscutível impacto na sobrevivência dos pacientes que evoluem em choque cardiogênico na fila de espera [3-6]. Este impacto pôde ser melhor avaliado a partir dos resultados do estudo REMATCH [13]. Este estudo comparou, de forma randomizada, os resultados do implante do dispositivo de assistência ventricular esquerda Heartmate com a evolução de pacientes mantidos clinicamente, abrindo a perspectiva do emprego definitivo deste tipo de terapêutica, como alternativa nos casos que apresentem contra-indicações ao transplante cardíaco.

O período de assistência proporcionado pelos diversos tipos de dispositivos pode variar de algumas semanas a vários meses, fato que depende de suas características específicas. Neste sentido, existem diversos tipos de dispositivos atualmente em uso clínico, sendo os mais comuns os ventrículos pneumáticos paracorpóreos e os ventrículos implantáveis de propulsão eletromecânica [4,5]. Os dispositivos paracorpóreos podem ser empregados na assistência à circulação sistêmica e pulmonar e apresentam um custo menor. A sua utilização, no entanto, exige a permanência dos pacientes em ambiente hospitalar. Já os ventrículos implantáveis podem apenas ser empregados na assistência ao coração esquerdo e apresentam um custo mais elevado. Por outro lado, permitem a manutenção dos pacientes em assistência por períodos superiores a um ano.
Resultados mais favoráveis com o emprego de dispositivos de suporte circulatório têm sido obtidos principalmente nos pacientes submetidos à assistência ventricular esquerda isolada [4,5,14], enquanto que os pacientes que necessitam assistência biventricular apresentam resultados limitados por causa dos maiores índices de complicações infecciosas e pulmonares [15,16]. Na cardiomiopatia chagásica, a disfunção biventricular e as arritmias ventriculares seriam fatores provavelmente responsáveis pela necessidade do emprego de assistência biventricular. Nos casos relatados na atual experiência, no entanto, foi possível a reversão da disfunção direita apenas com o suporte farmacológico no pós-operatório. Esta situação é freqüente em relação ao uso de dispositivos de assistência mecânica no tratamento de outras afecções [17] e mostra que a disfunção ventricular direita pode ser secundária ao comprometimento do coração esquerdo também na cardiomiopatia chagásica.

Outro aspecto importante observado nas diversas experiências relatadas na literatura é que a duração da assistência pode afetar significativamente o resultado do transplante. Pacientes mantidos em assistência por mais de 30 dias apresentam mortalidade hospitalar menor do que aqueles mantidos por menos de um mês [3]. Esse fato pode ser atribuído à reversão dos efeitos da insuficiência cardíaca sobre a função dos outros órgãos após a instalação da assistência, propiciando melhores condições para o transplante. Por outro lado, o contato do sangue com os biomateriais empregados nos dispositivos de assistência circulatória pode desencadear um aumento da resposta inflamatória, que envolve a ativação de sistemas de resposta celular e de proteínas plasmáticas [18]. Dessa maneira, o controle da biocompatibilidade dos sistemas artificiais assume grande importância para o sucesso do emprego destes dispositivos. Além das modificações empregadas nas superfícies de contato dos biomateriais, esse controle pode ser obtido pela inibição das cascatas biológicas que levam à ativação sangüínea e pelo controle dos produtos finais destas cascatas.

O dispositivo de assistência ventricular empregado neste estudo é um dispositivo paracorpóreo de desenvolvimento nacional, que tem a sua aplicação clínica vinculada a um protocolo de investigação aprovado após vários estudos "in vitro" e em animais de experimentação [19,20]. Nesta experiência, a manutenção dos pacientes por períodos que variaram de 11 a 31 dias, sem a ocorrência de complicações relacionadas ao próprio sistema de suporte circulatório, mantém aberta a perspectiva de seu emprego rotineiro em nosso meio. A este respeito, é importante se observar que o tempo médio de realização do transplante cardíaco para os pacientes à espera do doador em caráter de prioridade varia em nosso país entre um e dois meses, período que pode ser plenamente alcançado com o emprego 
de dispositivos paracorpóreos de assistência circulatória.

Em relação às complicações relatadas nesta experiência, no seguimento dos pacientes submetidos ao implante do dispositivo paracorpóreo de assistência ventricular InCor, é importante se destacar a necessidade de um acerto individual do protocolo de anticoagulação empregado nos primeiros casos da experiência. Esta situação se refletiu na elevada incidência de sangramento e de reoperações observada, a qual não comprometeu, no entanto, o resultado final do procedimento, à semelhança de outros relatos da literatura [21]. As outras complicações observadas são também freqüentemente relatadas na literatura [4,22], constituindo-se em aspectos que demonstram a complexidade do manuseio pós-operatório destes pacientes.

Como considerações finais desta experiência, podemos destacar que a cardiomiopatia chagásica é uma afecção cuja mortalidade será diminuída apenas com o implemento dos programas de transplante cardíaco, associado à disponibilidade de dispositivos de suporte circulatório mecânico. Nos pacientes chagásicos, o emprego destes dispositivos como ponte para o transplante cardíaco, à semelhança do que é realizado no tratamento de outras afecções, deve ser lembrado de maneira sempre precoce.

\section{REFERÊNCIAS BIBLIOGRÁFICAS}

1. Anyanwu AC, Rogers CA, Murday AJ. Intrathoracic organ transplantation in the United Kingdom 1995-99: results from the UK cardiothoracic transplant audit. Heart. 2002;87(5):449-54.

2. Tsao CI, Lin HY, Lin MH, Ko WJ, Hsu RB, Hwang SL et al. Influence of UNOS status on chance of heart transplantation and posttransplant survival. Transplant Proc. 2004;36(8):2369-70.

3. Deng MC, Edwards LB, Hertz MI, Rowe AW, Kormos RL. Mechanical Circulatory Support device database of the International Society for Heart and Lung Transplantation: first annual report-2003. J Heart Lung Transplant. 2003;22(6):653-62.

4. Proceedings of the 5th International Conference on Circulatory Support Devices for Severe Cardiac Failure. Ann Thorac Surg. 2001;71(3 suppl):S55-222.
5. Frazier $\mathrm{OH}$. Mechanical circulatory support: new advances, new pumps, new ideas. Semin Thorac Cardiovasc Surg. 2002;14(2):178-86.

6. Jessup M. Mechanical cardiac-support devices: dreams and devilish details. N Engl J Med. 2001;345(20):1490-3.

7. Bestetti RB. Predictors of unfavourable prognosis in chronic Chagas' disease. Trop Med Int Health. 2001;6(6):476-83.

8. Mady C, Cardoso RH, Barretto AC, da Luz PL, Bellotti G, Pileggi F. Survival and predictors of survival in patients with congestive heart failure due to Chagas' cardiomyopathy. Circulation. 1994;90(6):3098-102.

9. Bocchi EA, Fiorelli A. The paradox of survival results after heart transplantation for cardiomyopathy caused by Trypanosoma cruzi. First Guidelines Group for Heart Transplantation of the Brazilian Society of Cardiology. Ann Thorac Surg. 2001;71(6):1833-8.

10. Stolf NA, Higushi L, Bocchi E, Bellotti G, Auler JO, Uip D et al. Heart transplantation in patients with Chagas' disease cardiomyopathy. J Heart Transplant. 1987;6(5):307-12.

11. Marin-Neto JA, Marzullo P, Sousa AC, Marcassa C, Maciel $\mathrm{BC}$, Iazigi $\mathrm{N}$ et al. Radionuclide angiographic evidence for early predominant right ventricular involvement in patients with Chagas' disease. Can J Cardiol. 1988;4(5):231-6.

12. Bocchi EA, Vieira ML, Fiorelli A, Hayashida S, Mayzato M, Leirner A et al. Alterações hemodinâmicas e neurohormonais durante assistência circulatória com ventrículo artificial seguida de transplante cardíaco. Arq Bras Cardiol. 1994;62(1):23-7.

13. Rose EA, Gelijns AC, Moskowitz AJ, Heitjan DF, Stevenson LW, Dembitsky W et al. Long-term mechanical left ventricular assistance for end-stage heart failure. $\mathrm{N}$ Engl J Med. 2001;345(20):1435-43.

14. Sun BC, Catanese KA, Spanier TB, Flannery MR, Gardocki MT, Marcus LS et al. 100 long-term implantable left ventricular assist devices: the Columbia Presbyterian interim experience. Ann Thorac Surg. 1999;68(2):688-94.

15. El Banayosy A, Arusoglu L, Kizner L, Tenderich G, Boethig $\mathrm{D}$, Minami K et al. Predictors of survival in patients bridged to transplantation with the thoratec VAD device: a single-center retrospective study on more than 100 patients. J Heart Lung Transplant. 2000;19(10):964-8.

16. Farrar DJ, Hill JD, Pennington DG, McBride LR, Holman WL, Kormos RL et al. Preoperative and postoperative comparison of patients with univentricular and biventricular support with the thoratec ventricular assist device as a bridge to cardiac transplantation. J Thorac Cardiovasc Surg. 1997;113(1):202-9. 
17. Santamore WP, Austin EH, III, Gray L, Jr. Overcoming right ventricular failure with left ventricular assist devices. J Heart Lung Transplant. 1997;16(11):1122-8.

18. Baufreton C, Kirsch M, Loisance DY. Measures to control blood activation during assisted circulation. Ann Thorac Surg. 1998;66(5):1837-44.

19. Oshiro MS, Hayashida SA, Maizato MJ, Marques EF, Stolf NA, Jatene AD et al. Design, manufacturing, and testing of a paracorporeal pulsatile ventricular assist device: Sao Paulo Heart Institute VAD. Artif Organs. 1995;19(3):274-9.
20. Benício A, Moreira LFP, Hayashida S, Cestari IA, Leirner AA, Stolf NAG, Jatene AD. Avaliação do desempenho hemodinâmico do dispositivo de assistência ventricular Incor como substituto do coração esquerdo. Rev Bras Cir Cardiovasc. 1999;14(3):237-46.

21. Goldstein DJ, Beauford RB. Left ventricular assist devices and bleeding: adding insult to injury. Ann Thorac Surg. 2003;75(6 suppl):S42-7.

22. Holman WL, Rayburn BK, McGiffin DC, Foley BA, Benza $\mathrm{RL}$, Bourge RC et al. Infection in ventricular assist devices: prevention and treatment. Ann Thorac Surg. 2003;75(6 suppl.):S48-57. 